\title{
Discovery of Metal and Metalloid (Mg, V, Mn, Fe, Co, Ni, Cu, Zn, Se and Te) Chelators Produced in the Aerobic Anoxygenic Phototrophs
}

\author{
VLADIMIR YURKOV, STEVEN B KUZYK AND \\ ELIZABETH HUGHES
}

University of Manitoba

Presenting Author: umkuzyks@myumanitoba.ca

Aerobic anoxygenic phototrophs are Alpha-, Beta- and Gamma-Proteobacteria discovered few decades ago, which can only synthesize and utilize photosynthetic light harvesting systems in the presence of oxygen. They are incapable of anaerobic photosynthesis and photoautotrophy, distinguishing them from all other anoxygenic photosynthetic bacteria. Many of them possess exceptionally high resistance to oxyanions such as tellurite, tellurate, selenite and selenate and some were found to tolerate 2300 to $2700 \mu \mathrm{g} / \mathrm{ml}$ of $\mathrm{K}_{2} \mathrm{TeO}_{3}$, usually toxic to microbes at as low as $1 \mu \mathrm{g} / \mathrm{ml}$. Representatives have been isolated from a rich variety of environments including marine ecosystems, freshwater and meromictic lakes, hypersaline springs, and biological soil crusts, all in the hopes of understanding their ecological role and functions.

Over 100 isolates were chosen for this study, representing 44 species from 27 genera. They were tested for their interactions with iron and other metal cations such as $\mathrm{Mg}, \mathrm{V}, \mathrm{Mn}, \mathrm{Co}, \mathrm{Ni}, \mathrm{Cu}$, $\mathrm{Zn}$, Se and Te using a chromeazurol S assay to detect siderophore production. Representatives from 12 species in 8 genera of $\alpha$-Proteobacteria, or $21.8 \%$ of strains, produced highly diffusible siderophores that could bind one or more metals. In addition, $\gamma$-proteobacterial Chromocurvus halotolerans strain EG19, excreted a brown compound into growth medium, which was purified and confirmed to act as a siderophore. It had an approximate size of $341 \mathrm{Da}$ and drew similarities to the siderophore rhodotorulic acid, a member of the hydroxamate group, previously found only among yeasts. This study is the first to discover siderophore production to be wide spread among the aerobic anoxygenic phototrophs, which may be another key method of metal chelation and potentially detoxification within their environments. 